\title{
An Ant Colony Algorithm Model Construction on the Impact of Urban Real Estate Value and National Economic Changes
}

\author{
Suhong Liao \\ Jiangxi University of Finance and Economics, Nanchang, 330013, China \\ Correspondence should be addressed to Suhong Liao; 1202100001@jxufe.edu.cn
}

Received 24 November 2021; Revised 10 December 2021; Accepted 15 December 2021; Published 27 January 2022

Academic Editor: Tongguang $\mathrm{Ni}$

Copyright ( $) 2022$ Suhong Liao. This is an open access article distributed under the Creative Commons Attribution License, which permits unrestricted use, distribution, and reproduction in any medium, provided the original work is properly cited.

\begin{abstract}
With the continuous development of social economy, urban real estate has gradually become an important commodity for trading, and to some extent, it also affects the trend of the national economy. In view of these limitations and demand, this paper introduces ant group algorithm, by combining the influence of urban real estate changes on the national economic trend; according to the relevant national policy impact, we analyze the national economy changes and fluctuations, try to build a "fourquadrant" model analysis of real estate market impact analysis, and clear national policy on real estate. In order to obtain better economic policies, we promote the healthy and stable development of urban real estate. Simulation experiment proved that ant colony algorithm is effective and supports the change of urban real estate value and national economy.
\end{abstract}

\section{Introduction}

With the continuous development of social economy, urbanization is becoming more and more mature, promoting the transaction of urban real estate. Therefore, as an important trading commodity, it will also affect the trend of the national economy to some extent $[1,2]$. How to trace the urban real estate and macroeconomic changes is extremely important [3,4]. Since the reform and opening up, China has gradually formed a socialist market economy system. However, due to its national conditions, it is more special and complex, rather than a single factor affecting the real estate market. Therefore, it cannot be fitted with single linear influence factors $[5,6]$. Various economic crises in the world have a certain impact on China's economy. Although strong policies stimulate the economic development of domestic demand, it is still inevitably affected by the economic crisis; especially compared with the economic development of developed countries, there are still certain disadvantages and limitations $[7,8]$. How to influence the influencing factors of China's economy, especially for the satisfaction of export and internal demand, and how to achieve China's economic growth? How to effectively develop urban real estate which can truly develop healthy socialist market economy? These are all problems that need to be solved by industry experts, entrepreneurs, etc.

In view of these problems and demand, this paper introduces ant algorithm, by combining the socialist market economy development of real estate and using four quadrant for real estate market changes; according to the relevant national policy influence, we analyze the national economy changes and fluctuation and aim to explore the macroreal estate development and change, for the long-term equilibrium analysis of urban real estate market.

\section{Introduction of Ant Group Algorithm}

Ant colony algorithm can divide the real estate market accordingly, into the property market and the asset market, and use the corresponding real estate rent and the newly developed construction real estate to establish corresponding connections. The so-called first quadrant is that the property demand determines the corresponding level of real estate rent, the second quadrant represents the asset market, this part is to use the corresponding real estate for capital realization, and the third quadrant is to increase the corresponding amount of new development, to achieve a certain scale of growth. The fourth quadrant is the long-term 
conversion of the newly developed construction volume to real estate.

2.1. Image Segmentation Feature Extraction. The image segmentation algorithm was designed based on the ant colony algorithm [9-11]. By extracting the image segmentation features as the starting point, the element features required in the image are determined to design the path of the image segmentation.

In an image, including the target background in addition to the desired target, the image segmentation method is to segment the target from the target background. In the process of segmentation, the edge value and noise need to be set, which mainly ensures that the segmentation route of the target is intact and the target required to protect the image from damage. When setting the image segmentation feature, the pixel gray scale is selected as the first feature of the image segmentation, and the pixel gray scale can be used to distinguish the image background from the image target. Entering the pixel gray scale into the colony yields the corresponding values. Identifying the segmentation route and entering the neighborhood gray scale and gradient into the colony give the edge values for image segmentation. The edge values largely control the route and noise point of the image segmentation, so they take the gray scale value and the neighborhood gray scale value and gradient as the auxiliary to determine the segmentation characteristics of the image and input the corresponding segmentation value into the ant group algorithm.

\subsection{Build a Mathematical Model of the Ant Colony Search} Process. After determining the image segmentation feature, the segmentation feature needs to be input into the ant colony algorithm, so the mathematical model of the ant colony search process is constructed [12-15]. The mathematical model simulates the trajectory of the ant colony searching for food to calculate, which can accurately determine the immediate obstacles in the search process and ensure the accuracy of the calculation results. Set the initial image as $X$. Each pixel in $X$ forms a set. Set the set as $X_{j}$. The values of the elements in the set are not equal. Based on this, the travel path of ants is determined, and the image segmentation path is judged according to the process of ants looking for food.

The distance between $x_{i n}$ is arranged, and the distance between ant crawling is shown as

$$
d_{i j}=\sqrt{\sum_{k=1}^{m} p_{k}\left(x_{i k}-x_{j k}\right)^{2}},
$$

where $m$ represents the number of input image segmentation features. In this formula, the value of $m$ is set to $3 ; p_{k}$ represents the weighted value of the Euclidean distance, and the magnitude of this value depends on the degree of clustering influence of pixels on the image. The distance between each pixel can be obtained by this formula.
Set the clustering radius of the image to $r, p h_{i j}$ is the amount of information on the path of the ant colony, and determine the guiding function formula in the ant colony algorithm to obtain the guiding probability $p_{i j}$ :

$$
\begin{aligned}
p_{i j} & =\frac{r}{d_{i j}} \\
& =\frac{r}{\sqrt{\sum_{k=1}^{m} p_{k}\left(x_{i k}-x_{j k}\right)^{2}}},
\end{aligned}
$$

where the clustering radius $r$ is in the numerator and the denominator $d_{i j}$ is the distance, which is fixed. Therefore, the larger the clustering radius $r$, the larger the value of the guiding probability $p_{i j}$ of the guiding function, that is, the greater the probability of the ant choosing the path and vice versa. According to the guidance probability, $p_{i j}$ value of the guidance function is finally obtained; the ant's travel route is determined, so as to analyze the edge value of the image segmentation.

2.3. Set the Individual Ant Colony Rules. After completing the mathematical model of the colony search process, bootstrap rules were set against each colony for the calculated bootstrap probability values based on 3 features of the image segmentation [16-18].

2.3.1. Gray-scale Value Feature Rule. Gray-scale values are important features that distinguish the target in an image from the image background and can reduce a lot of computational workload when the gray-scale values in the image are determined. When using colony calculation, colonies can be arranged on the same starting point, the first gray-scale value. After then, the first gray-scale value is determined, the mathematical model calculation can be performed as described above, and the bootstrap probability return of the bootstrap function is determined based on the value of the pixel point.

2.3.2. Gradient Characteristic Rules. Gradient features are important features of analyzing critical and noise points, and different gradient images of the target and the background can be obtained according to the gradient, while also determining gradient pixels points in different ant colonies. Setting the number of pixels in different colonies to $n$, when $n$ is greater than the pixel mean, indicates that the region of the colony is a cluster center. The gradient feature of 0 is set in the cluster center of the image so that the cluster center of the image segmentation can be resolved.

2.3.3. Neighborhood Gray-Scale Value Feature Rule. Unlike gray-scale values, neighborhood gray-scale values are mainly used for the calculation of image segmentation edges, controlling the details of image segmentation and playing an important role in protecting image pixel work. The neighborhood gray-scale value is set to the regional form of $3 \times 3$; combined with the gradient characteristics above, the region 
with gradient characteristics to zero is set to the cluster center, and the neighborhood gray-scale value feature is set to 6 , so the corresponding field value can be obtained to determine the image segmentation direction.

2.4. Set Up the Initial Cluster Center. Setting the initial cluster center reduces a lot of computational work, where the colony moves within the specified range. When the initial cluster center is not set, the ant colonies will walk in the image irregularly, without guiding direction and guiding factors, resulting in excessive computational workload. Therefore, when setting the initial arrangement position of the colony, the initial cluster center should be determined, giving the colony a fixed region and reducing the search time.

When setting the initial cluster center, the cluster center may be located at the center or edge of the image and set according to the gradient characteristics of the image. At the edge of the target and background, the number of pixels is large and excessive pixels will result in the gradient value within the region being zero, so when setting the initial cluster center, the pixel number of the edge with the pixel value of the image target and the background as the starting point.

\subsection{Implement the Polymorphic Ant Colony Algorithm for} Image Segmentation. Through the setting of the initial cluster center, each ant colony is arranged in the cluster center. In the initial cluster center, the movement of the ant colony is irregular and blind. The distance between each pixel is measured, and the distance data $d_{i j}$ are obtained, and a mathematical model is constructed according to the length of the distance. In this model, the movement of the ant colony mainly uses pheromone to calculate the path, calculates and analyzes the probability according to the movement of the ant colony, and obtains the path of the ant colony through the obtained probability, so as to realize the image segmentation of the polymorphic ant colony algorithm.

\section{Principle of Macropolicy and Principle of Real Estate Industry}

The continuous development of our economy, the price of real estate is very obvious, especially for economic growth, domestic market domestic demand, and urban real estate as a new stimulus consumption growth point, and demand is very obvious, as shown in Figure 1; in the domestic real estate market, with the growth of the demand curve in the first quadrant of ant algorithm, the real estate rent level and demand will increase at the same time. In particular, for the increase of the rent level in the real estate market, the government has issued various policies, such as tax cuts and preferential ways to encourage real estate transactions, leading to an increase in the price of real estate assets. Therefore, macroeconomic growth will make the residential market develop diffuse, as shown in Figure 2.

However, in the actual development process, the real estate industry has experienced overheated real estate

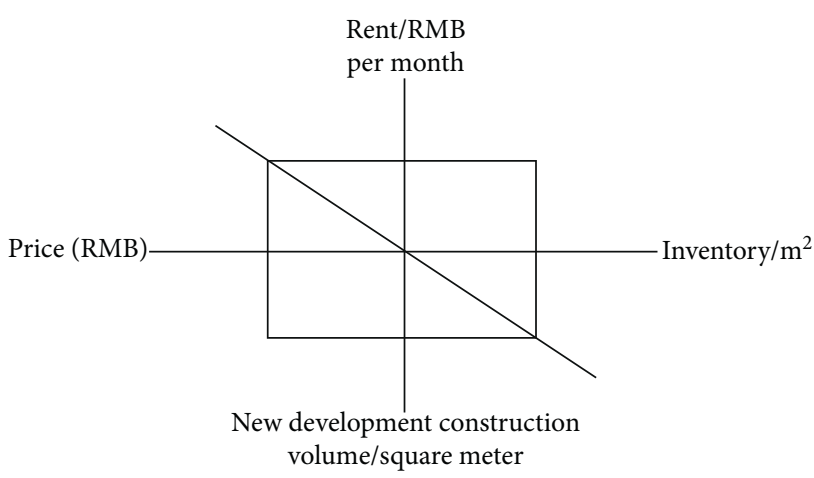

FiguRE 1: Four-quadrant pattern diagram.

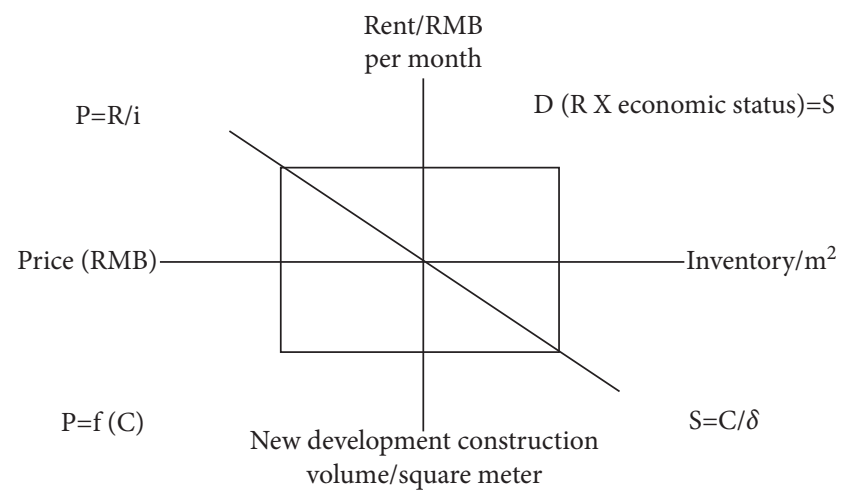

Figure 2: Four-quadrant pattern diagram.

investment in some areas. The prosperity of real estate has exceeded the development level of the local economy, showing the situation and characteristics of the mismatch with the economic development. Therefore, the state uses the corresponding policies, legal and administrative means to treat them differently, realizes the regulation of tax, interest rate, foreign investment, land, and other aspects, and realizes the adjustment of two angles of demand and supply.

3.1. From the Perspective of Control Requirements. First of all, the state and the government have issued a number of policies to conduct investment and trading rules and carried through a series of measures to conduct speculation in real estate and noninvestment demand. Through the corresponding ant colony algorithm and through the real estate improvement of loan and tax and the corresponding policy changes, the demand for real estate investment income has been improved. As shown in Figure 3, the improvement of different capitalization rates can bring about the corresponding equilibrium changes. When the expected development and construction amount is reduced, the property stock and rent of the related real property have increased. This is counterbalanced with the new amount of development and construction.

In the long run, the rent of real estate increases and the corresponding capitalization, so as to reduce the real estate price, reduces the development and construction volume of 


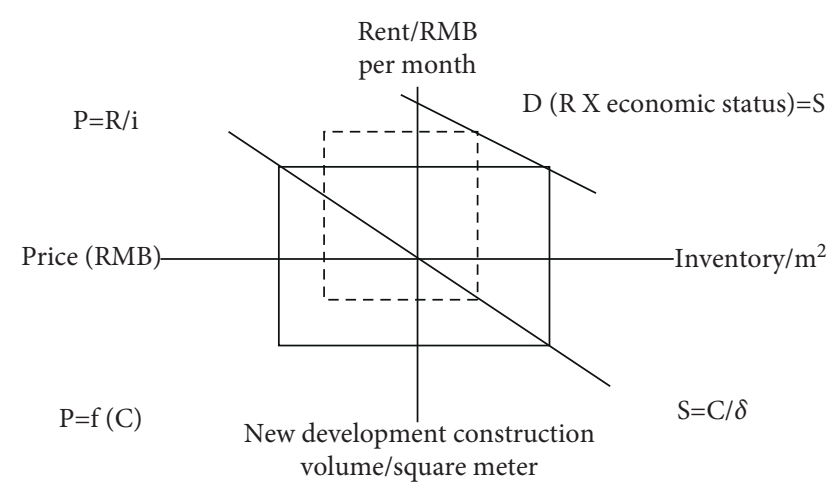

FIGURE 3: Four-quadrant pattern diagram.

the heart and stabilizes the real estate market. In the short term, the policy can reduce the demand for speculation to a certain extent, but due to the complexity of China's real estate market and the difference in regional development is too large, so the response of adjustment in real estate is too slow.

Equation (2) controls the supply.

From the perspective of supply control, it mainly covers credit, natural resources real estate, tax, etc. In terms of the ant colony algorithm, the land supply of the natural resource real estate, the control of the credit scale, and the real estate cost are reflected in the rays in Figure 4 to ensure that the balance state changes.

In the long run, these controlled supplies are in line with the long-term goal of the long-term control of the stable growth of China's real estate industry. In the short term, the real estate may have the supply ownership, but both the investment demands have been reduced. According to the corresponding theory of supply and demand relationship, the real estate price has been reduced and changed in a certain period of time, which means that the government can effectively control the housing price.

Through the ant group algorithm, in the case of the supply relationship changes to a certain extent, the real estate combining has been retained, and the use demand of real estate can meet different real estate ownership, and the rent will be relatively reduced. If the balance state changes and the demand curve is shifted, the speculative and nonspeculative demand for real estate will be reduced. When equilibrium is reached, the ant colony algorithm overall shrinks, contrary to economic expansion, as shown in Figure 5:

National macrocontrol policies range from control to protection, with the focus on supporting residents in solving housing problems.

The contribution rate is the specific monitoring and measurement of the overall impact of a certain index on the economy:

$$
\mathrm{Con}_{\mathrm{it}}=\frac{\Delta \mathrm{Val}_{\mathrm{it}}}{\Delta \mathrm{GDP}_{t}} \times 100 \% .
$$

From the calculation results, from 2018 to 2020, the contribution rate of the primary industry was on the rise, the

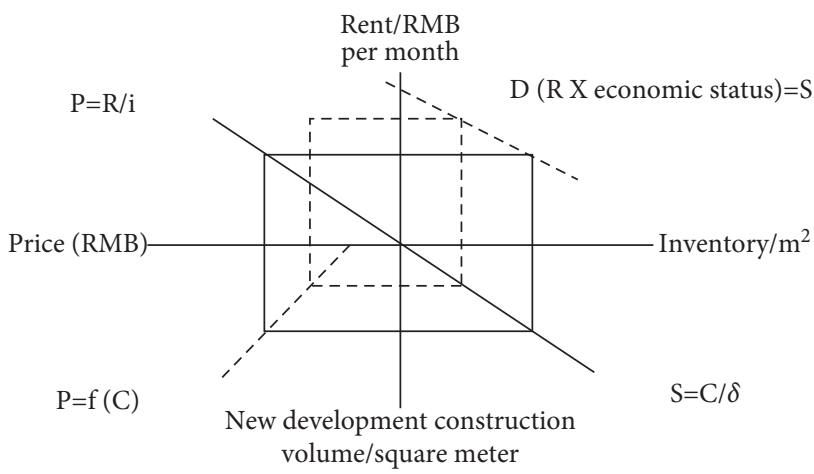

FIgURE 4: Four-quadrant pattern diagram.

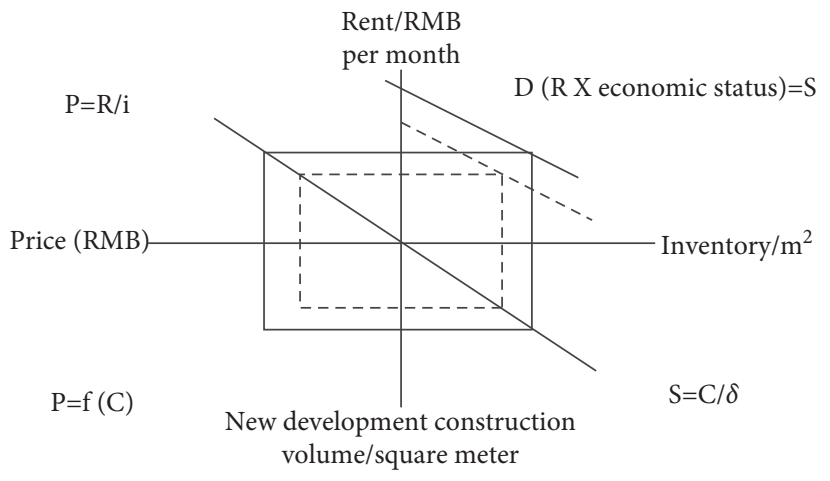

FIgURE 5: Four-quadrant pattern diagram.

contribution rate of the secondary industry was decreased, and the contribution rate of the tertiary industry showed a steady rise.

From the results in Figure 6, if the secondary industry declines, from the perspective of real estate alone, the economic contribution rate of real estate is basically about $7 \%$, but there is still a big gap compared with the contribution of industrial economy to China's economic growth.

\section{Simulation Experiment and Analysis}

4.1. Analysis Based on State Space Models. State space models are a powerful tool for the dynamic analysis of interrelations between variables. The model uses a powerful iterative algorithm called the Kalman filter to incorporate unobservable variables into the observable model and obtains estimates with it:

$$
\begin{aligned}
& \left\{\begin{array}{l}
\log \left(\mathrm{GDP}_{t}\right)=\alpha_{t}+\beta_{t} \log \left(\text { industry }_{t}\right) \times+\widehat{u} \\
\beta_{t}=a+b \times \beta_{t-1}+\widehat{\varepsilon}_{t}
\end{array}, \quad t=1,2, \ldots, T,\right. \\
& \left\{\begin{array}{l}
\log \left(G D P_{t}\right)=\alpha_{t}^{\prime}+\beta_{t}^{\prime} \log \left(\text { realestate }_{t}\right) \times+\widehat{u}^{\prime} \\
\beta_{t}^{\prime}=a^{\prime}+b^{\prime} \times \beta_{t-1}^{\prime}+\widehat{\varepsilon}_{t}^{\prime}
\end{array}, \quad t=1,2, \ldots, T .\right.
\end{aligned}
$$

Using the data provided by the China Statistical Yearbook 2020, the changes in the parameters $\beta_{t}$ and $\beta_{t}^{\prime}$ of the sample period can be obtained, as shown in Figure 7 . The 


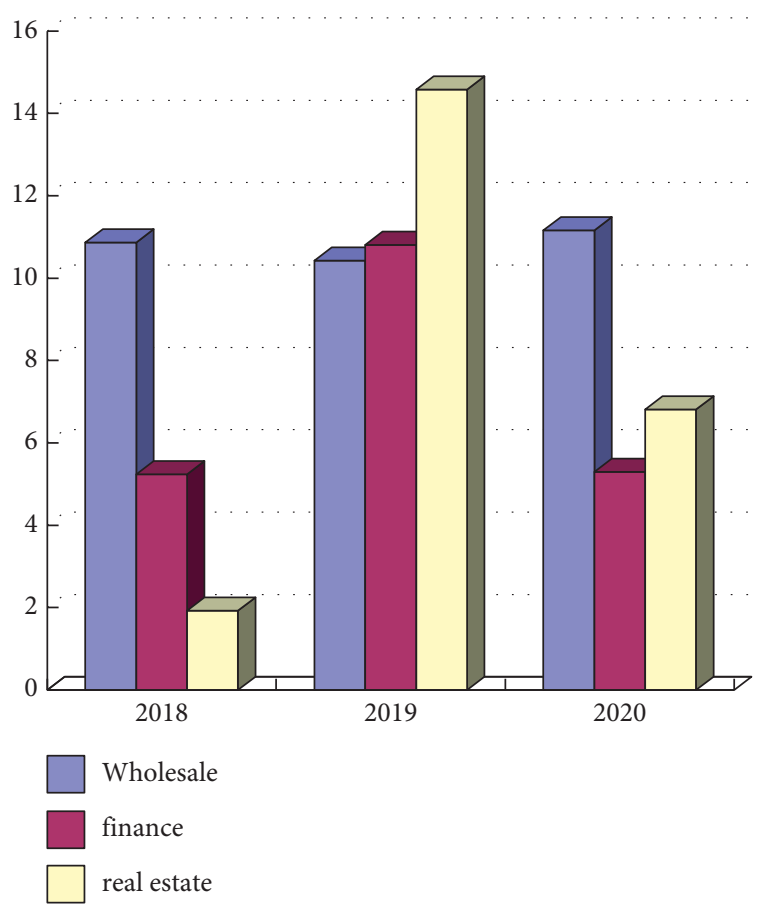

Figure 6: Industry contribution unit (\%) from 2018 to 2020.

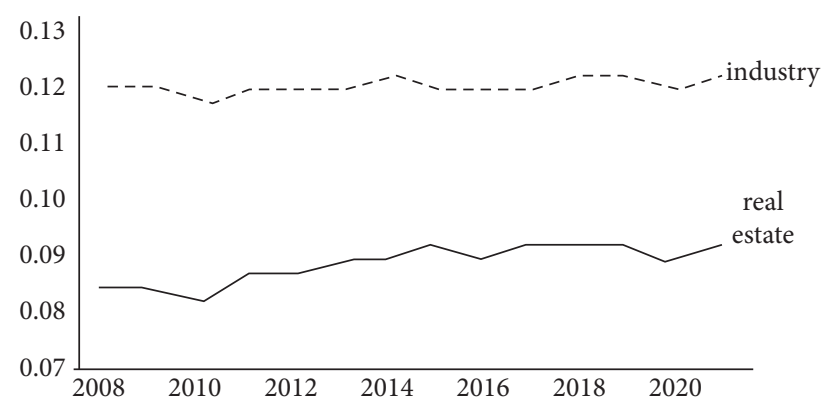

Figure 7: Parameter changes of the state space model from 2002 to 2015.

elasticity of GDP to industry is relatively stable, fluctuating around 0.12 , while in the same period, although the elasticity of GDP to real estate shows the upward trend, the tertiary industry represented by real estate cannot yet replace the position of the secondary industry.

4.2. To Absorb Employment. From the perspective of urban employment personnel, among the 20 industries of China's national economy, the person directly engaged in real estate accounts for not much, only accounting for about $2 \%$.The top three industries are manufacturing, construction, and education, accounting for $27.7 \%, 15.3 \%$, and $9.5 \%$, respectively, 12.1 times, 6.7 times, and 4.1 times the number of real estate practitioners, respectively (Figure 8).

As a capital company, the capital party, its purpose is to obtain operating profit. In the general concept, the real estate development is good, its operating profit is relatively high,

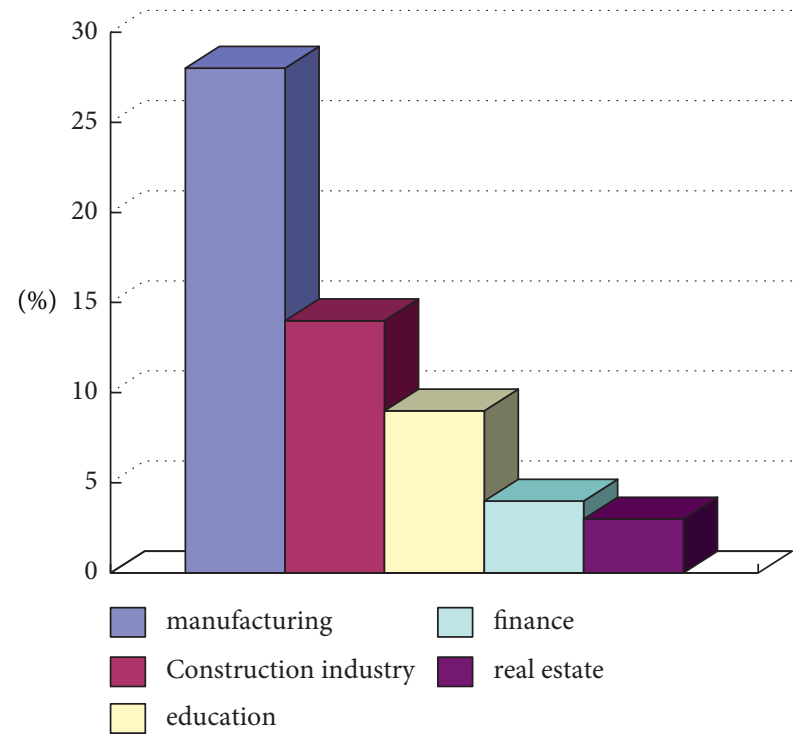

Figure 8: Employment of urban units in some industries accounted for units (\%) in 2020.

the real estate development is not good, and its operating profit is relatively low. Although China's real estate market has basically maintained a bull market for more than ten years, the real estate industry profit should be a lot.

As a capitalist real estate company, the purpose of its operation is to obtain operating profits. In the general concept, if real estate develops well, its operating profit is relatively high. If real estate develops well, its operating profit is relatively low. China's real estate market has basically maintained a dozen or so despite occasional fluctuations. In the bull market of 2015, the real estate industry should have made a lot of profits.

According to China's current division of powers and financial powers, local governments undertake a large number of tasks, but financial powers are concentrated in higher-level governments, making local governments have to regard real estate as the "second fiscal" in order to complete economic and social development tasks. Real estate has a huge impact on local finances. To resolve the dependence of local governments on real estate development, it is necessary to solve the dependence of local finance on real estate development. Only by liberating local finance from land gains can the local government's benign guiding role in real estate development be fundamentally given play to. Reform the existing fiscal and taxation system and reasonably divide central and local affairs so that local governments have sufficient financial resources to effectively handle their affairs without relying on land and provide guarantees for local economic development, people's lives, and social stability.

The issue of human capital has always been a key area of economic research. In the history of economic research, Malthus' theory of population disasters has appeared, and the important role of human capital in economic growth has also been strongly promoted. Despite the school disputes, it is undeniable that human capital is an important factor in a country's economic growth. Empirical evidence shows that 
there is a close correlation between population growth and economic growth. The recession of the Japanese economy, the stagnation of the European economy, the arrival of an aging society, and the negative growth of the population appeared. As the world's most populous country, China's most basic resource is human capital. In 1978, China implemented reform and opening up. The low labor cost is one of the important reasons why the economy can develop in great strides. In addition to the large population, another important reason for the low labor cost in China is that China's family planning policy started in the last century has caused the dependency ratio to drop drastically and enjoy a huge demographic dividend. However, the current population aging in China is very fast. In 2015, the number of people over 60 years old in China exceeded 200 million, accounting for $16.1 \%$, and the number of people over 65 years old has reached 140 million, accounting for 10.5\%. According to the aging society standard that the population over 60 years old accounted for more than $10 \%$ and the population over 65 years old accounted for more than $7 \%$, China has actually entered an aging society, and the aging degree will be further increased in recent decades. The heavier burden and the decline in the proportion of the right-age labor force have greatly increased the average cost of labor in our country and have brought tremendous pressure to the normal operation of enterprises and the normal operation of individual families. For China, maintaining the sustainable development of the economy and society is still the biggest task in the future period of history. It is recommended to further adjust the family planning policy to promote the moderate growth of the population and continue to maintain the competitiveness of China's enterprises and the sustainable development of the family.

The tax burden of economic units in China is relatively heavy. The measurement caliber of the macrotax burden has three categories: narrow caliber, medium caliber, and wide caliber. The narrow caliber refers to the proportion of tax revenue in GDP, the medium caliber refers to the proportion of fiscal revenue in GDP, and the wide caliber refers to the proportion of revenue that all governments can control or influence in GDP. China's narrow-caliber macrotax burden is less than $20 \%$, the medium-caliber macrotax burden is slightly higher than $20 \%$, and the wide-caliber macrotax burden is close to $40 \%$. From the perspective of the relationship between households, enterprises, and the country and the development of households and enterprises, a widecaliber macrotax burden is more reasonable. At present, China's wide-caliber macrotax burden has been much higher than the average level of developing countries and even higher than the level of some developed countries. The burden of taxes and fees makes the economic unit's operating results unable to be used for technological innovation and self-development, and the development of competitiveness is greatly affected. For China's sustainable development, tax cuts are an important way to mobilize the enthusiasm of households and enterprises and stimulate their creativity. In the future, necessary explorations should be made in tax cuts, especially structural tax cuts.
Science and technology are primary productive forces. Since the reform and opening up, China has gradually increased its investment in science and technology research and development, and the progress in science and technology has made rapid progress, which has promoted the rapid development of China's economy and has become the world's second largest economy. However, despite the rapid progress in science and technology in China, the gap with developed countries is still huge. The intuitive manifestation of the relatively weak technological innovation ability is the low added value of technology made in China. Technology is the main source of the added value of high-tech products, and the price of commodities is the most important competitiveness of non-high-tech products, but the price factor is greatly affected by the cost. In the context of reduced labor, higher tax costs, and rising raw material prices, the competitiveness of China's low value-added products has dropped significantly. In the future, we should optimize the technological innovation environment, increase investment in science and technology, improve China's technological innovation capabilities, especially the technological innovation capabilities of enterprises, and then increase the technological added value of Chinese products so that the advantages of Chinese products will be transformed from low prices to advanced technology.

\section{Conclusions}

With the continuous development of social economy, urban real estate has an influence on the macroeconomy, and how to trace its external influence factors is very important. In view of these limitations and needs, this paper is based on ant algorithm, by combining the operation of the real estate market to adjust the real estate market, using national policy impact analysis, explore the national policy on real estate, to effectively adjust the policy, using the "four-quadrant" model to analyze the impact of the real estate market, further promote the healthy development of real estate, and realize the effective development of national economy. Simulation experiments have proved that the ant colony algorithm is effective.

\section{Data Availability}

The labeled dataset used to support the findings of this study is available upon request to the author.

\section{Conflicts of Interest}

The author declares no conflicts of interest.

\section{Acknowledgments}

This work was supported by Jiangxi University Humanities and social sciences research project: Research on Three Effects and Policy Optimization of the Impact of Housing Wealth Change on Residents' Economic Behavior, project approval no. jj21106. 


\section{References}

[1] M. S. Andersen, G. Levin, and M. V. Odgaard, "Economic benefits of reducing agricultural $\mathrm{N}$ losses to coastal waters for seaside recreation and real estate value in Denmark," Marine Pollution Bulletin, vol. 140, no. 3, pp. 146-156, 2019.

[2] Q. Chen, S. M. Kamran, and H. Fan, "Real estate investment and energy efficiency: evidence from China's policy experiment," Journal of Cleaner Production, vol. 217, no. 20, pp. $440-447,2019$.

[3] S. Hu and Q. Li, "Modeling and simulating a smart information-based real estate online platform," Procedia Computer Science, vol. 111, no. 4, pp. 339-347, 2017.

[4] S. J. Petheram, A. C. Nelson, M. Miller, and R. Ewing, "Use of the real estate market to establish light rail station catchment areas: case study of attached residential property values in salt lake county, Utah, by light rail station distance," Transportation Research Record, vol. 5, no. 6, pp. 1-9, 2018.

[5] V. Alcántara and E. Padilla, "CO2 emissions of the construction sector in Spain during the real estate boom: input-output subsystem analysis and decomposition," Journal of Industrial Ecology, vol. 5, no. 9, pp. 109-117, 2021.

[6] G. Giustiziero, "Is the division of labor limited by the extent of the market? opportunity cost theory with evidence from the real estate brokerage industry," Strategic Management Journal, vol. 4, no. 6, pp. 176-184, 2020.

[7] D. Higgins and T. Perera, "Advancing real estate decision making: understanding known, unknown and unknowable risks," International Journal of Building Pathology and Adaptation, vol. 36, no. 4, pp. 373-384, 2018.

[8] B. Alireza, "Integrating value and uncertainty in the energy retrofit analysis in real estate investment-next generation of energy efficiency assessment tools," Energy Efficiency, vol. 8, no. 5, pp. 1015-1034, 2015.

[9] L. Jian, M. K. Kang, and C. L. Lee, "Transportation infrastructure improvement and real estate value: impact of level crossing removal project on housing prices," Transportation, vol. 48 , no. 1 , pp. $1-8,2021$.

[10] F. A. F. Ferreira, R. W. Spahr, M. A. Sunderman, and M. S. Jalali, "A prioritisation index for blight intervention strategies in residential real estate," Journal of the Operational Research Society, vol. 69, no. 8, pp. 178-185, 2018.

[11] V. D. Pinto and A. M. Rinaldi, "A configurational approach based on geographic information systems to support decisionmaking process in real estate domain," Soft Computing, vol. 5, no. 1, pp. 1-9, 2018.

[12] S. Nazlioglu, N. Alper-Gormus, and U. Soytas, "Oil prices and real estate investment trusts (REITs): gradual-shift causality and volatility transmission analysis," Energy Economics, vol. 65 , no. 1 , pp. 19-27, 2016.

[13] E. Akalpler and S. Hove, "Carbon emissions, energy use, real GDP per capita and trade matrix in the Indian economy-an ARDL approach," Energy, vol. 168, no. 1, pp. 1081-1093, 2019.

[14] J. Guo, H. He, and J. Peng, "Real-time energy management for plug-in hybrid electric vehicle based on economy driving pro system," Energy Procedia, vol. 158, no. 7, pp. 2689-2694, 2019.

[15] X. Zhang, K. Shu, S. Rajkumar, and V. Sivakumar, "Research on deep integration of application of artificial intelligence in environmental monitoring system and real economy," Environmental Impact Assessment Review, vol. 86, no. 1, pp. 17-25, 2020.

[16] G. An, C. Becker, and E. Cheng, "Housing price appreciation and economic integration in a transition economy: evidence from Kazakhstan," Journal of Housing Economics, vol. 52, no. 3, pp. 101-118, 2021.

[17] F. Ullah, S. M. E. Sepasgozar, M. Jamaluddin-Thaheem, and F. Al-Turjman, "Barriers to the digitalisation and innovation of Australian Smart Real Estate: a managerial perspective on the technology non-adoption," Environmental Technology \& Innovation, vol. 3, no. 1, pp. 101-107, 2021.

[18] G. An, C. Becker, and E. Cheng, "Bubbling away: forecasting real estate prices, rents, and bubbles in a transition economy," Comparative Economic Studies, vol. 63, no. 3, pp. 1-8, 2021. 